\title{
Comparative Evaluation of Disinfecting Alginate Using Aloe Vera and $0.2 \%$ Chlorhexidine Digluconate by Internal Disinfection Method - An In Vivo Study
}

\author{
Tanu Mahajan ${ }^{1}$ \\ ${ }^{1}$ Department of Prosthodontics and Dentistry, Rama Dental College and Hospital, Kanpur, Uttar Pradesh, India.
}

\section{ABSTRACT}

\section{BACKGROUND}

Aloe vera and chlorhexidine are traditionally used as disinfectants of irreversible hydrocolloids. Most popular among irreversible hydrocolloids is alginate which is most commonly used in dentistry. We wanted to compare and evaluate the efficacy of aloe vera and $0.2 \%$ chlorhexidine as an internal disinfectant.

\section{METHODS}

For each subject three impressions of the upper arch were made using irreversible hydrocolloid impression material i.e. Alginate (Zelgan 2002). After the impression was made, a sterile cotton swab was used and wiped over the impression surface from the center of the palate and was sent for culture sensitivity test. This swab acted as the control group (Group 1). The swab was then placed in normal saline transport medium and was sent for culture sensitivity test to the Gyan Pathology Lab, Kanpur. The swab was then run over the Blood Agar culture media. Incubation of the culture medium plates was done at $37^{\circ} \mathrm{C}$ for $24 \mathrm{hrs}$. From the culture plate, smear was taken to examine bacterial growth on a slide, semi-quantitatively, under light microscope, and the colony forming units were then counted. 24 hours after initial impression, second impression (Group 2) was made by mixing alginate with 99.9\% aloe vera. 48 hours after the first impression, the third impression (Group 3) of the same subject was made after mixing alginate with $0.2 \%$ chlorhexidine digluconate. Remaining procedural steps were similar for second and third impressions as the first impression. Bacterial count was recorded and analysed for variation between group 1, group 2 and group 3 using Repeated Measures ANOVA with post-hoc Bonferroni test from SPSS version 18.

\section{RESULTS}

This study showed that using 99.9\% concentration of Aloe Vera as an internal disinfectant to manipulate irreversible hydrocolloid impression material did show a reduction $(65.96 \%)$ in growth of aerobic bacteria. However, the decrease was significantly less when compared with chlorhexidine.

\section{CONCLUSIONS}

Amongst the two disinfecting agents used in this study, $0.2 \%$ chlorhexidine digluconate has shown to be more effective disinfecting agent than Aloe Vera.

\section{KEY WORDS}

Aloe Vera, Antibacterial Activity, Chlorhexidine, Disinfectant
Corresponding Author:

Dr. Tanu Mahajan,

117/54, L-Block,

Naveen Nagar,

Kanpur-208025,

Uttar Pradesh, India.

E-mail: drtanumahajan_27@yahoo.co.in

DOI: $10.14260 /$ jemds/2020/423

How to Cite This Article:

Mahajan T, Comparative evaluation of disinfecting alginate using aloe vera and $0.2 \%$ chlorhexidine digluconate by internal disinfection method- an invivo study. J. Evolution Med. Dent. Sci. 2020;9(27): 1944-1947, DOI:

10.14260/jemds/2020/423

Submission 10-02-2020,

Peer Review 04-06-2020,

Acceptance 10-06-2020,

Published 06-07-2020.

Copyright (C) 2020 JEMDS. This is an open access article distributed under Creative Commons Attribution License [Attribution 4.0 International (CC BY 4.0)] 


\section{BACKGROUND}

Infection control is an important aspect of dentistry. Dental impressions can become contaminated after removal from the patients mouth ${ }^{1}$ which can cross-infect casts which are poured against them. ${ }^{2}$ This Possibility of transfer of pathogens from dental surgery to dental laboratory is a matter of concern to the profession. ${ }^{3}$ Irreversible hydrocolloid is the most popular impression material used in dentistry owing to its acceptable elastic properties, comfort to the patient, ease with which it can be manipulated and its cost effectiveness. Alginates are hydrophilic in nature and due to variation in osmotic potential when they are immersed in liquids mean they readily imbibe water, which causes them to swell. The osmotic potential is reversed when watersoluble salts present in the alginate matrix are eluted, causing water to diffuse out and subsequent shrinkage of the material. These processes can take place simultaneously, so alginates become especially susceptible to distortion when immersion disinfection procedures are employed. Due to this property the accuracy of impressions is compromised, which can cause adverse effect on clinical accuracy of the resultant prosthesis fabricated on the casts made from these impressions. Irreversible hydrocolloid being partly organic, hydrophilic, irregular and porous in nature provides for retention and growth of microorganisms. ${ }^{4}$

Aloe vera has been used medicinally for a few thousand years and also finds vivid use in dentistry as well. Amongst the various species of aloe vera that are available the one with medicinal value is Aloe vera Barbadenis. ${ }^{5}$ The main advantage of using aloe vera as a disinfectant is that it is a natural product with no or minimal side effects, easily available and most importantly being 100 percent biodegradable, does not cause any harm to the environment.

Chlorhexidine has antibacterial and antifungal activities with broad-spectrum coverage. It is a cationic agent. It is also known to be compatible with oral mucosa. Chlorhexidine also has a unique property of substantivity, which means that it has the ability to remain on a particular surface and then be released in a gradual manner.6,7 Chlorhexidine digluconate is is used in a dilution of $0.12-0.2 \%$ as a mouthwash. It is a chemical antiseptic. Innumerable studies have found it to have a significant anti-plaque action. Studies done in the past have also revealed it to be especially acting against Gramnegative rods. It some conditions it is also used to prevent the occurrence of dental caries as an adjunctive treatment and also to treat periodontal disease. ${ }^{8}$ Research in past has proved the antibacterial efficacy of various chemical agents like chlorhexidine, povidone iodine, sodium hypo chloride, quaternary ammonium etc on irreversible hydrocolloid impression material. Aloe Vera gained our attention due to its growing popularity in the field of medicine and dentistry. Aloe Vera has been considered to be a miraculous herb with antibacterial, anti-viral and anti-inflammatory properties. It is a naturally growing herb, which is easily available, cheap and biodegradable with no significant harmful effects so far.

\section{Materials Required}

1. Aloe vera (99.96\% Pure, Patanjali Ayurved).

2. Chlorhexidine $(0.2 \%$ chlorhexidine digluconate $)$.

3. Alginate (Zelgan 2002).
4. Mouth mirror, probe and kidney tray.

5. Disposable glasses.

6. Disposable gloves and mouth mask

7. Perforated dentulous stock trays.

8. Rubber bowl.

9. Curved spatula.

10. Measuring scoop and cylinder.

11. Sterile cotton swabs.

12. Blood agar culture media.

13. Light microscope.

14. Incubator.

\section{METHODS}

This is an in-vivo study conducted in the Faculty of dental sciences, Department of Prosthodontics at Rama Dental College and hospital, Kanpur. A total of thirty subjects were selected from student volunteers of Rama Dental College, Kanpur. Patients with healthy mouth and all teeth present were included for the study and those with habit for pan chewing, smoking, drinking alcohol; completely edentulous, undergoing orthodontic or periodontal treatment, appliances present in the oral cavity and patient having any systemic disease were not included in the study.

For each subject three impressions were made of the maxillary arch using irreversible hydrocolloid impression material i.e. Alginate (Zelgan 2002). The subjects were made to sit upright on the dental chair. Conventional sterilization protocols were followed. Autoclaved impression trays were used to make the impressions. For First impression alginate was mixed with distilled water. The rubber bowl and the curved spatula were disinfected before alginate mixing. Alginate was dispensed in a clean rubber bowl by using scoop provided by the manufacturer and a measuring cylinder was used to dispense the water in a water powder ratio as recommended by the manufacturer. The alginate was mixed using a curved spatula in a figure of eight motion to achieve a smooth creamy consistency and loaded onto the impression tray and the impression was made. After the impression was made, a sterile cotton swab was used and wiped over the impression surface from the center of the palate and was sent for culture sensitivity test. This swab acted as the control group (Group 1). The swab was then placed in normal saline transport media and was sent for culture sensitivity test to the Gyan pathology lab, Kanpur. The swab was then run over the Blood Agar culture media. For 24 hrs. culture-medium plates were incubated at $37^{\circ} \mathrm{C}$. Semi-quantitative bacterial growth was examined of a smear taken from the culture plate on a slide under light microscope and the colony forming units were then counted. After 24 hours of making the initial impression, second impression (Group 2) was made by mixing alginate with $99.9 \%$ aloe vera. Then for the same subject, after 48 hours, third impression (Group 3) was made after the first impression, with Alginate mixed with $0.2 \%$ chlorhexidine digluconate. Rest of the procedural steps were same for both the second and third group of impressions as the first impression. 
RESULTS

Group 1 (alginate mixed with tap water) showed highest bacterial count when observed under light microscope while group 2 (alginate mixed with $99.9 \%$ aloe vera) showed less bacterial count, followed by group 3 (alginate mixed with $0.2 \%$ chlorhexidine). Although aloe vera is good disinfectant but results with chlorhexidine were much better. Even Some cultured samples of group 3 showed no growth of microorganisms.

\section{Statistical Analysis}

SPSS version 18 was used for all the analysis. A p-value of $<0.05$ was considered statistically significant. Log transformation of CFU (Colony Forming Unit) counts was done to ensure normality in distribution. Mean comparison was used with using Repeated Measures ANOVA with posthoc Bonferroni test.

\begin{tabular}{|lcccccccc|}
\hline \multicolumn{4}{|c}{ Group 1 } & \multicolumn{3}{c}{ Group 2 } & \multicolumn{3}{c|}{ Group 3 } & P-Value & Post-Hoc \\
Mean & SD & Mean & SD & Mean & SD & P-Vst \\
Log & 8.45 & 0.98 & 2.86 & 0.56 & 1.66 & 1.15 & $\begin{array}{c}<0.001 ; \\
\text { Sig }\end{array}$ & $1>2>3$ \\
CFU & \multicolumn{1}{|l}{ Table 1. Repeated Measures ANOVA with Post Hoc- Bonferroni Test } \\
\hline
\end{tabular}

\section{Interpretation}

Table 1 shows mean colony growth of aerobic bacteria in CFU in control i.e. Group 1 and the disinfected impressions i.e group 2 and group 3. Log conversion of CFU has been done to ensure normality in distribution. Growth was seen even after disinfection, however there was a mean reduction of growth when comparison was done between group 1, group 2 and group 3 using post- hoc test wherein group 3 showed least growth.

\begin{tabular}{|cc|}
\hline Intra-Group & P-Value \\
Group 1 vs 2 & $<0.001$; Sig \\
Group 1 vs 3 & $<0.001$; Sig \\
Group 2 vs 3 & $<0.001$; Sig \\
\hline Table 1a. Comparison between Group 1, Group 2 and Group 3 \\
Using Post Hoc Test \\
\hline
\end{tabular}

\section{Interpretation}

There was an overall significant difference in the mean CFU among the three groups. Post-hoc test showed that group 1 had significantly higher mean CFU than group 2 and 3 . Similarly, Group 2 had significantly higher mean CFU than 3.

\begin{tabular}{|ccc|}
\hline Percentage Reduction in Comparison to Control & Mean & SD \\
$\begin{array}{c}\text { Percent reduction with Group 2 } \\
\text { (Alginate mixed with 99.9 \% Aloe vera) }\end{array}$ & 65.96 & 7.25 \\
$\begin{array}{c}\text { Percentage reduction with Group 3 } \\
\text { (Alginate mixed with 0.2 \% Chlorhexidine digluconate) }\end{array}$ & 80.43 & 13.87 \\
\hline Table 2. Percentage Reduction in Comparison to Control & \\
\hline
\end{tabular}

\section{Interpretation}

Table 2 shows percentage reduction compared to control of group 2 and group 3 wherein group 3 shows significant decrease of $80.43 \pm 13.87 \%$ from group 1 and group 2 shows a significant decrease of $65.96 \pm 7.25 \%$ from group 1 .

\section{DISCUSSION}

The results show that there is about $80.43 \%$ reduction in growth of bacteria when $0.2 \%$ Chlorhexidine digluconate is used to mix alginate instead of water and about $65.96 \%$ reduction when Aloe Vera is used. This clearly depicts that $0.2 \%$ chlorhexidine digluconate markedly reduces the bacterial growth of aerobic culture when used as an internal disinfectant. Although Aloe Vera does have an antibacterial effect when used to mix alginate instead of water but when compared with $0.2 \%$ chlorhexidine digluconate, it is a weaker disinfectant. The disinfectant has dual role to fulfil, the first is that it should have effective antimicrobial property, and yet it should cause no adverse effect on the accuracy and surface details and also the texture of the impression material. In this study it has been observed that instead of water a disinfectant solution can be used to mix alginate which is found to be more effective than disinfecting the wateralginate impressions by immersion or spray disinfection ${ }^{3}$. This is a unique method wherein the disinfectant is incorporated in the impression mixture and thus all the problems associated with surface disinfection such as syneresis and imbibition are eliminated. ${ }^{9}$ Use of disinfectantsupplemented irreversible hydrocolloid impression materials when compared with the other disinfection techniques has proved to be more effective. Flanagan et al ${ }^{10}$ did a study to test the antimicrobial potential of alginate with added disinfectants. In total five groups were made. In two groups, no disinfectants were added and in the remaining, chlorhexidine and quaternary ammonium compounds were added. In the study five test microorganisms were included. It was seen that the quaternary ammonium-containing test group alginate was completely effective against all five test microorganisms. The test group alginate containing chlorhexidine was effective against all the gram-negative bacilli and the up-to $95 \%$ to $99 \%$ of the gram-positive cocci and yeast. However, in those test groups of alginates in which disinfectants were not added showed no antimicrobial effects. ${ }^{10}$

Thus, literature proves that using chemical agent like chlorhexidine digluconate instead of water to manipulate irreversible hydrocolloid impression is an effective measure to reduce cross contamination through impression. In this study, using $0.2 \%$ of chlorhexidine digluconate as an internal disinfectant to manipulate alginate while mixing has shown significant decrease $(80.43 \%)$ of growth of aerobic bacteria however the decrease was significantly less when compared with chlorhexidine.

\section{CONCLUSIONS}

Aloe Vera when used as an internal disinfectant has shown to have anti-bacterial properties reducing about $65.96 \%$ of bacterial growth. $0.2 \%$ Chlorhexidine Digluconate when used as an internal disinfectant has shown to have anti-bacterial 
properties reducing about $80.43 \%$ of bacterial growth. Amongst the two disinfecting agents used in this study, $0.2 \%$ chlorhexidine digluconate has shown to be more effective disinfecting agent than Aloe Vera.

Thus, it can be concluded that internal disinfection of alginate is an effective method for reducing the bacterial count to a great extent. Further studies should be taken up which can include even greater number of test pathogens. The added advantage of internal disinfection is that the impression can be poured immediately without waiting for the steps of disinfection and thus saving time and simultaneously maintaining accuracy.

\section{REFERENCES}

[1] Chau VB, Saunders TR, Pimsler M, et al. In-depth disinfection of acrylic resins. J Prosthet Dent 1995;74(3):309-13.

[2] Leung RL, Schonfeld SE. Gypsum casts as a potential source of microbial cross-contamination. J Prosthet Dent 1983;49(2):210-11.

[3] Rosen M, Touyz LZ. Influence of mixing disinfectant solution into alginate on working time and accuracy. J Dent 1991;19(3):186-8.
[4] Muzaffar D, Braden M, Parker S, et al. The effect of disinfecting solutions on the dimensional stability of dental alginate impression materials. Dent Mater 2012;28(7):749-55.

[5] Atherton P. How does aloe vera work? In: Atherton P, ed. The essential aloe vera. $2^{\text {nd }}$ edn. Newport: Mills Enterprises 1997: p. 35.

[6] Ullsfoss BN, Ogaard B, Arends J, et al. Effect of a combined chlorhexidine and NaF mouthrinse: an in vivo human caries model study. Scand J Dent Res 1994;102(2):109-12.

[7] Walters TH, Furr JR, Russel AD. Antifungal action of chlorhexidine. Microbios 1983;38(153-154):195-204.

[8] Scully C. Oral and maxillofacial medicine: the basis of diagnosis and treatment. $3^{\text {rd }}$ edn. Edinburgh: Churchill Livingstone 2013:39-41.

[9] Sevedge SR, Gunderson RB, Singer MT. Linear distortion and compatibility of an antimicrobial irreversible hydrocolloid impression material and improved dental stones. J Prosthet Dent 1989;62(5):612-5.

[10] Flanagan DA, Palenik CJ, Setcos JC, et al. Antimicrobial activities of dental impression materials. Dent Mater 1998;14(6):399-4. 